\title{
Invited Talk: Computational Persuasion with Applications in Behaviour Change
}

\author{
Anthony Hunter \\ Department of Computer Science, \\ University College London, \\ London, UK \\ anthony.hunter@ucl.ac.uk
}

\begin{abstract}
Persuasion is an activity that involves one party trying to induce another party to believe something or to do something. It is an important and multifaceted human facility. Obviously, sales and marketing is heavily dependent on persuasion. But many other activities involve persuasion such as a doctor persuading a patient to drink less alcohol, a road safety expert persuading drivers to not text while driving, or an online safety expert persuading users of social media sites to not reveal too much personal information online. As computing becomes involved in every sphere of life, so too is persuasion a target for applying computer-based solutions. An automated persuasion system (APS) is a system that can engage in a dialogue with a user (the persuadee) in order to persuade the persuadee to do (or not do) some action or to believe (or not believe) something. To do this, an APS aims to use convincing arguments and counterarguments in order to persuade the persuadee. Computational persuasion is a new field for the study of formal models of dialogues involving arguments and counterarguments, of user models, and strategies, for APSs. A promising application area for computational persuasion is in behaviour change. In this talk, I will review ongoing funded project ${ }^{1}$ being undertaken in the UCL Intelligent Systems Group on developing a framework for computational persuasion for behaviour change technology $[1,2]$.
\end{abstract}

\section{References}

1. Hunter, A.: Computational persuasion with applications in behaviour change. In: Computational Models of Argument (COMMA'16). pp. 5-18. IOS Press (2016)

2. Hunter, A.: Towards a framework for computational persuasion with applications in behaviour change. Argument and Computation (2018), in press

\footnotetext{
${ }^{1}$ For more information, see www.computationalpersuasion.com
} 